DOI 10.1007/s00167-005-0015-4

\author{
Roland M. Biedert \\ Silvia Albrecht
}

\section{The patellotrochlear index: a new index for assessing patellar height}

Received: 8 March 2005

Accepted: 25 May 2005

Published online: 23 February 2006

(c) Springer-Verlag 2006
R. M. Biedert $(\bowtie) \cdot$ S. Albrecht Swiss Federal Institute of Sports, Orthopaedics \& Sport Traumatology, University of Basle, 2532 Magglingen, Switzerland

E-mail: bi@scvl.ch

Tel.: + 41-32-3276490

Fax: + 41-32-3276405

\begin{abstract}
The radiological methods to determine patellar height described in the literature are variable, not reliable and depend on the chosen ratio. The purpose of this paper is to describe another method of measuring patellar height on sagittal MRI using the true articular cartilage patellotrochlear relationship. An analysis of magnetic resonance (MR) examinations of 66 consecutive patients was performed. The most common diagnoses were meniscal or anterior cruciate ligament pathologies. No patient suffered from patellofemoral complaints. Measurements on sagittal MR images included different parameters using the articular cartilage of the patella and the trochlea. The ratio patella : trochlea of the cartilage baselines was measured in percentages and described as patellotrochlear index. The measurements were assessed at two different times by three raters under blinded conditions. The mean patellotrochlear
\end{abstract}

index was $31.7 \%$ (CI: 12.5-50.0; range -5.0 to $61.1 \%$; $\mathrm{SD} \pm 11.6$ ). The intraobserver variability showed only in the "second observer" a difference of the mean values of the two different measurements $(t=2.189$; $P=0.032)$. The interobserver correlation was high and significant (0.663-0.893; $P=0.000)$. Our results indicate that the patellotrochlear index is a reliable and precise method to determine the exact articular correlation of the patellofemoral joint and the patellar height. The results represent the average patellotrochlear index in the normal population without patellofemoral complaints. Measurements of the articular cartilage congruence can be helpful to define an underlying pathology of patellar height, such as patella alta or infera.

Keywords Patellar height Measurement - MRI ·

Articular cartilage $\cdot$ Patellofemoral

\section{Introduction}

The term patella alta is commonly used in the literature $[2,7,18,21,22,23,25,29,31]$. It is mentioned with regard to patellar instability $[10,12,19,20,26,27,32]$, pain $[2,11,15,17,31]$, chondromalacia $[18,22]$, gonarthrosis [1, 24], ossifications in the patellar tendon [8], and adolescent growth spurt [23]. Therefore patella alta is considered a relevant pathologic factor in patellofemoral disorders. Currently, the definition of patella alta is insufficient and not clear [18, 21], although numerous methods to measure patellar height have been described $[3,5,9,13,16]$. Seil et al. [28] showed that patellar height classification and patella alta depend heavily on the chosen ratio. The differing results were mainly due to the anatomical (bony) differences [28].

The methods to determine patella alta or, in general patellar height, described in the literature are not reliable $[21,28]$. In reality these methods measure the length of the patellar tendon to the longest sagittal diameter of the 
patella ratio (Insall and Salvati [16]), the length of the patellar tendon to the length of the articular surface of the patella (Grelsamer et al. [14]), the length of the articular surface of the patella to the height of the lower pole of the articular surface above a tibial plateau line (Blackburne and Peel [5]), the distance between the distal point of the patellar articular surface and the anterosuperior border of the tibia divided by the length of the articular surface of the patella (Caton et al. [9]), and the distance between the superior line of the trochlea and the inferior edge of the articular surface of the patella (Bernageau et al. [3]). All these different measuring methods use lateral radiographs to determine patellar height where the articular cartilage is not visible. Bosshard et al. [6] showed that there is a significant difference in the articular cartilage joint geometry and the corresponding subchondral osseous anatomy of the patella, and the femoral trochlea [30]. This means that all ratios using radiographs do not measure the real articular congruence between patella and distal femur but measure ratios given by different and variable osseous landmarks.

We think that the only ratio that is accurate for the determination of patellar height and for all patients who are present with a clinical disorder and some complaint about patellofemoral pain is the ratio between the articular cartilage of the patella and the trochlear cartilage. This study aimed (1) to determine the patellotrochlear articular cartilage ratio (2) in a normal population, to define patella alta, normal, and patella infera, and (3) to present this patellotrochlear index as another method to measure patellar height.

\section{Materials and methods}

Patients

We analyzed the MR examinations of sixty-six consecutive patients. Most of the patients had meniscal or anterior cruciate ligament pathologies. No patient suffered from patellofemoral complaints, neither anamnestic nor during the physical examination.

\section{MR measurements}

MR examinations were performed using a Magnetom Impact 1.0 Tesla (Siemens) with the knees in $0^{\circ}$ of flexion, the foot in $15^{\circ}$ external rotation, and the quadriceps muscle consciously relaxed. Measurements on sagittal MR images included different parameters [4] (Figs. 1, 2): (1) Baseline patella $\left(\mathrm{BL}_{\mathrm{P}}\right)$; (2) Baseline trochlea $\left(\mathrm{BL}_{\mathrm{T}}\right)$; (3) Ratio $\mathrm{BL}_{\mathrm{P}}: \mathrm{BL}_{\mathrm{T}}$. The parameters were measured on the midline sagittal section MRI through the patella with the thickest articular cartilage and maximal length of the patellar bone. The patellotrochlear index was calculated as the Baseline patella $\left(\mathrm{BL}_{\mathrm{P}}\right)$ :Baseline trochlea $\left(\mathrm{BL}_{\mathrm{T}}\right)$ ratio measured in percentages.

The measurements on the MR images were assessed independently at two different times (more than a sixweek interval) by three raters (the two authors and another orthopaedic surgeon) who were blinded to the patients' data.

\section{Statistical analysis}

For statistical analysis we used the total index in percentages. Paired samples $t$ test was performed to examine intraobserver variability. Spearman's rank correlation was calculated to determine the interobserver correlation. To determine "normal values" of the patellotrochlear index we used the $95 \%$ confidence interval (lower/upper bound). A $P$ value of 0.05 was considered to be significant. Statistical analysis was performed with Statistical Package of Social Sciences (SPSS).

\section{Results}

The sixty-six patients (forty-four males, twenty-two females) had an average age of 38.2 years (range, 1236 years). The mean patellotrochlear ratio was $31.7 \%$ (CI: $12.5-50.0$; range -5.0 to $61.1 \%$; $\mathrm{SD} \pm 11.6$ ). The paired samples $t$ test showed only in the "second observer" a difference of the mean values of the two different measurements $(t=2.189 ; \quad P=0.032)$. The interobserver correlation was high and significant $(0.663-0.893 ; P=0.000)$.

\section{Discussion}

Standard radiographs document only the osseous contours of the patellofemoral joint and the position of the patella in reference to the trochlea. Thickness and condition of the articular cartilage can only be measured using MRI or MR arthrotomography. Bosshard et al. [6] found no contour congruence of articular cartilage surface and subchondral bone of the patellofemoral joint both in the sagittal and axial plane. Our study confirms the significant cartilage-bone mismatch in the sagittal plane of the patellofemoral joint. The osseous anatomy in the sagittal plane of the patellofemoral joint does not correspond with the articular cartilage surface. We agree with Seil et al. [28] that the most important factor in patellar height determination is the position of the articular surface of the patella in relation to the trochlear cartilage (Fig. 3a, b). Statements about patella alta or infera, using only standard radiographs, must therefore be interpreted with caution and awareness of this fact. 


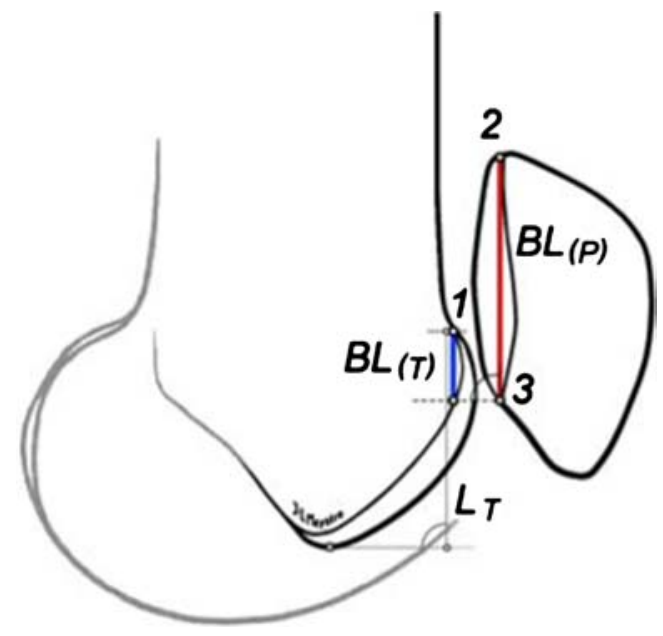

Fig. 1 Patellotrochlear index measurement ${ }^{4} .: B L_{P}$ Baseline patella (2 superior most aspect of articular cartilage to 3 inferior most aspect); $B L_{T}$ : Baseline trochlea (length of trochlear articular surface from 1 superior most aspect with respect to 3 the inferior most aspect of the articular patellar cartilage using a right angle and parallel lines); Ratio $B L_{T} B L_{P}$ calculated in percentages; $L_{T}$ Length of trochlear cartilage (superior most aspect to inferior most aspect of trochlea using a vertical line)

With regard to the statistical analysis $(95 \%$ confidence interval; lower/upper bound), we think that index values of more than $50 \%$ document patella infera and less than $12.5 \%$ document patella alta.

The described patellotrochlear index measured on sagittal MR images is a reliable and reproducible

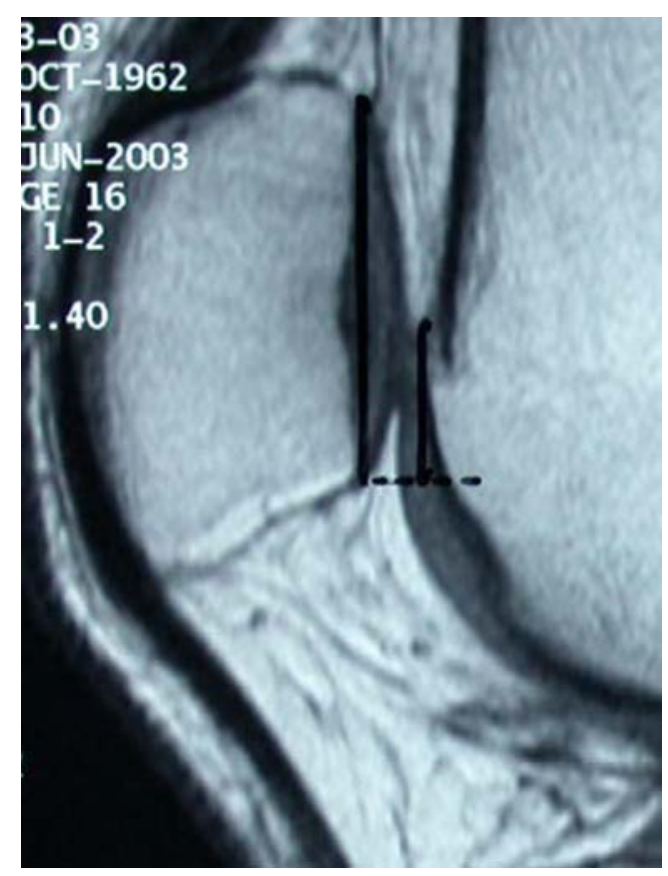

Fig. 2 Measurement of the patellotrochlear index on sagittal MRI

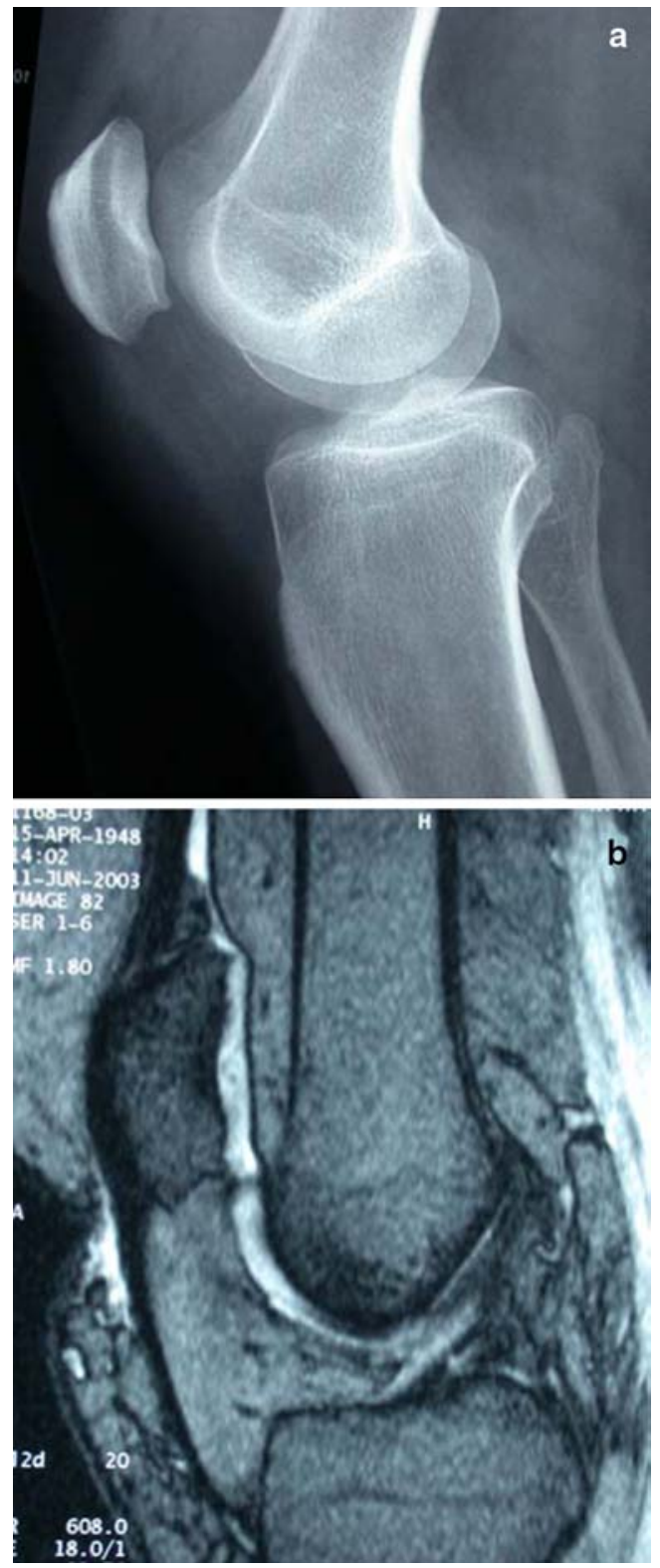

Fig. 3 a Sagittal radiograph showing normal radiological patellar height ratios measured according to Blackburne-Peel [5], InsallSalvati [16], Grelsamer [13], Caton-Deschamps [9]. b MR measurement of the same patient showing no contact of the patellar and trochlear articular cartilage. The patellotrochlear index is $0 \%$ documenting patella alta

method to determine the exact articular correlation of the patellofemoral joint and the patellar height. The results of our study showed that the inter-and intraobserver variability is low which was confirmed by low mean standard errors and high correlation coefficients.

The major advantages of this index are: (1) exact measurement of the patellotrochlear articular congruence; (2) osseous form variations of the patella (i.e. long, 


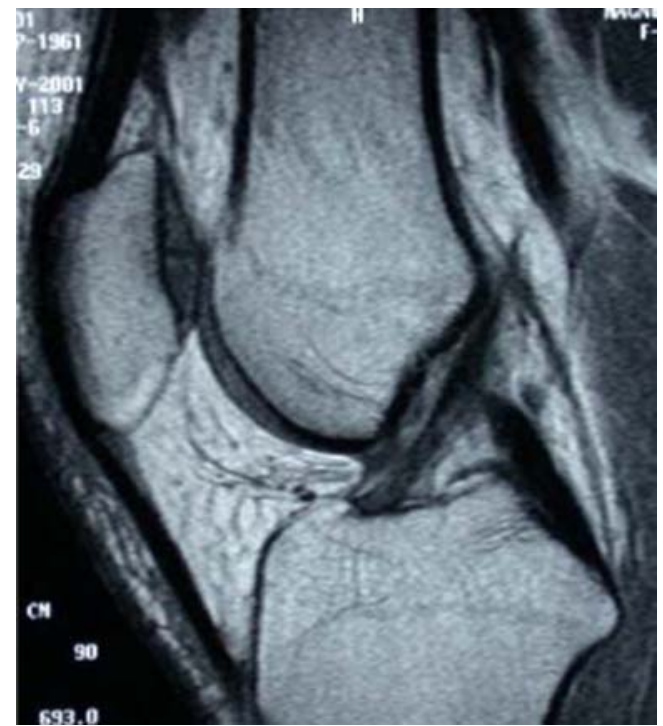

Fig. 4 Sagittal MR shows long and flat trochlea, long sagittal osseous diameter of the patella (nose) with relatively short articular cartilage

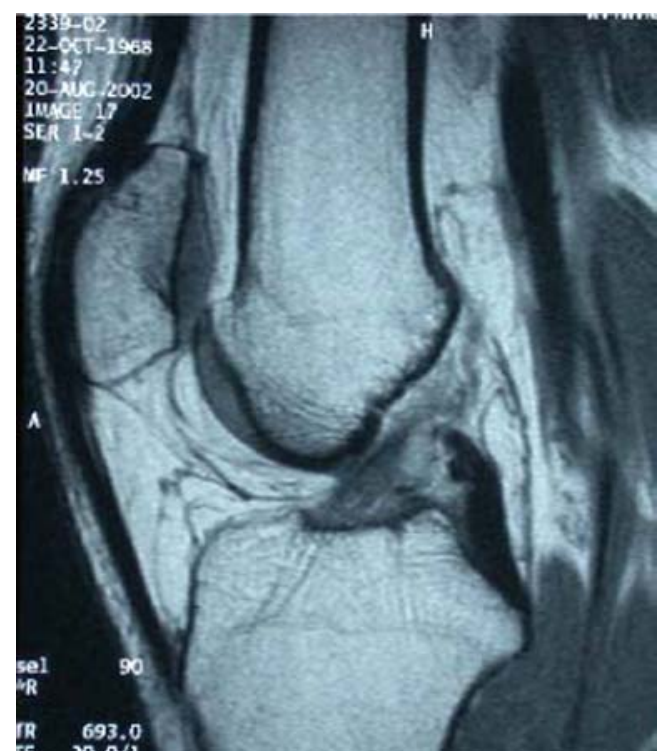

Fig. 5 Sagittal MR shows short trochlea and relatively short osseous sagittal diameter of the patella compared to the articular cartilage

nonarticular inferior pole) [14] do not affect the ratio (Figs. 4, 5), (3) differences of length and shape of the trochlea are considered; (4) variations of the patellar tendon attachment areas are insignificant (Sinding-Larson-Johansson or Osgood-Schlatter disease; after surgical interventions); (5) thicker radiolucent cartilage in children is visible; (6) no ionizing radiation exposure, and (7) measurements in $0^{\circ}$ knee flexion is easier than in $30^{\circ}$ of flexion.
In contrast to Miller et al. [25], we did not measure the trochlear articular length using the distal femoral physeal scar. Our evaluation of the MRI examination documented that this point of reference lays frequently more proximal than the end of the trochlear cartilage, shows variations, and is therefore not as precise as our described method (Fig. 6a, b). In our opinion, the most superior aspect of the trochlear articular surface can be determined easier and more precisely than the distal femoral physeal scar. In addition, the articular cartilage provides more evidence for the articular congruence than the physeal scar.
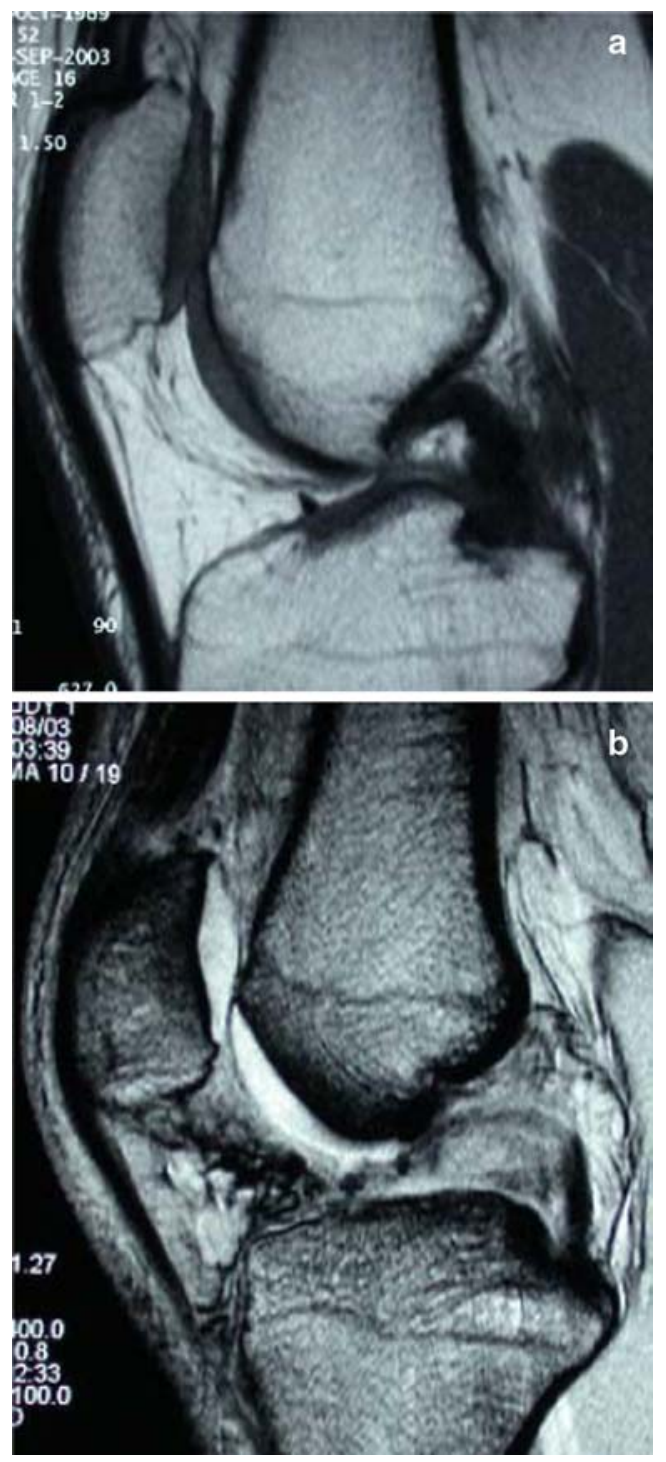

Fig. 6 The distal femoral physeal scar does not correspond with the superior most aspect of the trochlear articular cartilage. Note also the variations of form and length of the trochlear cartilage. a Physeal scar too distal and raising anteriorly. b Physeal scar too proximal 
If abnormal patellar height (alta or infera) is considered to be the underlying pathology in the patellofemoral joint causing instability or pain, then an MRI is recommended, especially if conservative treatment was not successful, and surgery may be considered. The patellotrochlear index describes the real articular cartilage relationship. Sagittal MRI might therefore be helpful to plan precisely any distalization or proximalization of the patella. Additional axial views show the condition and real congruence of the articular cartilage, and all types of trochlear dysplasia may be depicted.

In conclusion, the described patellotrochlear index documents the individual's real articular cartilage relationship in the patellofemoral joint and represents another method to measure precisely patellar height.
The presented technique of measurement has also limitations. First, the control of conscious quadriceps muscle relaxation was difficult (no artifacts on the images were accepted). Second, no weight bearing was possible. Third, imaging in greater flexion angles $\left(30^{\circ}\right.$ or $60^{\circ}$ ) has not yet been performed for comparison. Fourth, no repeat MRIs were done on the same patient. Further studies are needed to assess: (1) the reliability of this measurement on successive MRIs in the same patient, (2) how the amount of quadriceps contraction influences this reading, and (3) whether this reading has any validity to the patellofemoral pathology.

\section{References}

1. Ahlback S, Mattsson S (1978) Patella alta and gonarthrosis. Acta Radiol 19:578-584

2. AL-Sayyad MJ, Cameron JC (2002) Functional outcome after tibial tubercle transfer for the painful patella alta. Clin Orthop 396:152-162

3. Bernageau J, Goutallier D, Debeyre J, Ferrrane J (1969) Nouvelle technique d'exploration de l'articulation fémoropatellaire. Incidences axiales quadriceps contracté et décontracté. Rev Chir Orthop 61(Suppl II):286-290

4. Biedert RM, Albrecht S (2003) The patellotrochlear index: another method of measuring patellar height on sagittal MRI. In: Proceedings of the international patellofemoral study group, Naples FL, USA

5. Blackburne JS, Peel TE (1977) A new method of measuring patellar height. J Bone Joint Surg Br 59:241-242

6. Bosshard C, Stäubli HU, Rauschning W (1997) Konturinkongruenz von Gelenkoberflächen und subchondralem Knochen des Femoropatellargelenks in der sagittalen Ebene. Arthroskopie 10:72-76

7. Brattstrom H (1970) Patella alta in nondislocating knee joints. Acta Orthop Scand 41:578-588

8. Bruijn JD, Sanders RJ, Jansen BR (1993) Ossification in the patellar tendon and patella alta following sports injury in children. Complications of sleeve fractures after conservative treatment. Arch Orthop Trauma Surg 112:157-158
9. Caton J, Deschamps G, Chambat P, Lerat JL, Dejour H (1982) Patella infera. A propos of 128 cases. Rev Chir Orthop Reparatrice Appar Mot 68:317

10. Dejour H, Walch G, Nove-Josserand L, Guier C (1994) Factors of patellar instability: an anatomic radiographic study. Knee Surg Sports Traumatol Arhrosc 2:19-26

11. Dupont JY (1998) Patellofemoral pain. Rev Prat 48:1781-1786

12. Geenen E, Molenaers G, Martens M (1989) Patella alta in patellofemoral instability. Acta Orthop Belg 55:387393

13. Grelsamer RP, Meadows S (1992) The modified Insall-Salvati ratio for assessment of patellar height. Clin Orthop 282:170-176

14. Grelsamer RP, Proctor CS, Bazos AN (1994) Evaluation of patellar shape in the sagittal plane: a clinical analysis. Am J Sports Med 22:61-66

15. Hirano A, Fukubayashi T, Ishii T, Ochiai N (2001) Relationship between the patellar height and the disorder of the knee extensor mechanism in immature athletes. J Pediatr Orthop 21:541544

16. Insall J, Salvati E (1971) Patella position in the normal knee joint. Radiology 101:101-104

17. Kannus PA (1992) Long patellar tendon: radiographic sign of patellofemoral pain syndrome - a prospective study. Radiology 185:859-863

18. Karadimas JE, Piscopakis N, Syrmalis L (1981) Patella alta and chondromalacia. Int Orthop 5:247-249

19. Kujala UM, Osterman K, Kvist M, Aalto T, Friberg O (1986) Factors predisposing to patellar chondropathy and patellar apicitis in athletes. Int Orthop 10:195-200
20. Lancourt JE, Cristini JA (1975) Patella alta and patella infera. Their etiological role in patellar dislocation, chondromalacia, and apophysitis of the tibial tubercle. J Bone Joint Surg Am 57:1112-1115

21. Leung YF, Wai YL, Leung YC (1996) Patella alta in southern China. A new method of measurement. Int Orthop 20:305-310

22. Marks KE, Bentley G (1978) Patella alta and chondromalacia. J Bone Joint Surg Br 60:71-73

23. Micheli LJ, Slater JA, Woods E, Gerbino PG (1986) Patella alta and the adolescent growth spurt. Clin Orthop 213:159-162

24. McWilliams TG, Binns MS (2000) A locked knee in extension: a complication of a degenerate knee with patella alta. J Bone Joint Surg B 82:890

25. Miller TT, Staron RB, Feldman F (1996) Patellar height on sagittal MR imaging of the knee. Am J Roentgenol 167:339-341

26. Neyret $\mathrm{P}$, Robinson AH, Le Coultre B, Lapra C, Chambat P (2002) Patellar tendon length - the factor in patellar instability? Knee 9:3-6

27. Rillmann P, Dutly A, Kieser C, Berbig R (1998) Modified Elmslie-Trillat procedure for instability of the patella. Knee Surg Sports Traumatol Arthrosc 6:31-35

28. Seil R, Muller B, Georg T, Kohn D, Rupp S (2000) Reliability and interobserver variability in radiological patellar height ratios. Knee Surg Sports Traumatol Arthrose 8:231-236

29. Simmons E Jr, Cameron JC (1992) Patella alta and recurrent dislocation of the patella. Clin Orthop 274:265-269 
30. Stäubli HU, Bosshard C, Porcellini B, Rauschning W (2002) Magnetic resonance imaging for articular cartilage: cartilage-bone mismatch. Clin Sports Med 21:1-17
31. Topoleski TA, Kurtz CA, Grogan DP(2000) Radiographic abnormalities and clinical symptoms associated with patella alta in ambulatory children with cerebral palsy. J Pediatr Orthop 20:636639
32. Walker P, Harris I, Leicester A (1998) Patellar tendon-to-patella ratio in children. J Pediatr Orthop 18:129-131 